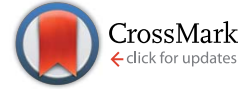

Cite this: RSC Adv., 2016, 6, 25789

Received 14th January 2016 Accepted 1st March 2016

DOI: $10.1039 / c 6 r a 01154 c$

www.rsc.org/advances

\section{An investigation of surface properties, local elastic modulus and interaction with simulated pulmonary surfactant of surface modified inhalable voriconazole dry powders using atomic force microscopy}

\author{
Sumit Arora, ${ }^{\text {abc }}$ Michael Kappl, ${ }^{b}$ Mehra Haghi, ${ }^{c d}$ Paul M. Young, ${ }^{c}$ Daniela Traini ${ }^{\mathrm{c}}$ \\ and Sanyog Jain*a
}

L-Leucine is used as the most common force control agent used in the inhaled dry powder formulations. In this study, the effect of L-leucine on the surface morphology, surface energy and the Young's modulus of the composite spray dried particles was studied. In addition, how L-leucine modifies the interaction of particles with a simulated pulmonary surfactant is also studied. Voriconazole (VRZ) was spray dried with different concentrations of L-leucine from hydroalcoholic solutions. Formulations were found to possess irregular morphology. Surface concentration of L-leucine was increased with increasing feed concentration and plateaued at about $20 \% \mathrm{w} / \mathrm{w}$ L-leucine. Atomic force microscopy (AFM) coupled with drug colloid probe enabled measurement of cohesion forces between the prepared formulations and found cohesion to be reduced significantly $(p<0.05)$ with the increase of L-leucine concentration. Peak Force Tapping enabled characterization of nanomechanical properties (elasticity and deformation) of formulations. Co-spray drying L-leucine with VRZ does not seem to have any influence on the Young's modulus of the formulations. Lastly, AFM revealed that the surface chemistry of the drug particle and pulmonary surfactant, as well as the contact geometry of the interacting surfaces, plays an important role in determining nature and extent of interaction between inhaled drug particles and pulmonary surfactant.

\section{Introduction}

The lung represent an attractive portal for the delivery of drugs not only for the treatment of local pathological conditions but also for the systemic delivery of drugs, proteins and peptides in order to exert systemic pharmacological actions. ${ }^{1,2}$ Traditionally, pressurized metered dose inhalers (pMDIs) have been the device of choice for the aerosolized delivery of drugs. Low dose delivery and use of propellants are some of the drawbacks of pMDIs which led to innovations in other pulmonary delivery systems. ${ }^{3}$ Dry powder inhalers are of particular interest to pharmaceutical research and industry owing to their ability to deliver large doses of drug, ease of administration, short

${ }^{a}$ Centre for Pharmaceutical Nanotechnology, Department of Pharmaceutics, National Institute of Pharmaceutical Education and Research (NIPER), Sector 67, S.A.S. Nagar, Mohali, Punjab-160062, India.E-mail: sanyogjain@niper.ac.in; sanyogjain@ rediffmail.com; Fax: +91-172-2214692; Tel: +91-172-2292055

${ }^{b}$ Max Planck Institute for Polymer Research, 55128 Mainz, Germany

${ }^{\circ}$ Respiratory Technology, Woolcock Institute of Medical Research and Discipline of Pharmacology, Sydney Medical School, The University of Sydney, NSW 2037, Australia ${ }^{d}$ Faculty of Pharmacy, Graduate School of Health, University of Technology Sydney, NSW 2007, Australia operation time and portability, all leading to improved patient compliance. ${ }^{3,4}$

The primary consideration for the inhaled drug particles is their aerodynamic particle size should lie in the range of 1-5 $\mu \mathrm{m}$. Powders in this size range generally exhibit highly cohesive behavior and a tendency to aggregate limiting their utility as inhalable dry powder formulations. ${ }^{5,6}$ Hence, it is imperative to control particle cohesion so as to improve the dispersibility of dry powder formulations.

There are literature reports which have shown that co-spray drying L-leucine with the drugs resulted in dry powders with decreased agglomeration and superior aerosolization efficiency rendering them suitable for pulmonary administration. The effect of L-leucine in limiting the cohesiveness of developed formulations is often being explained by its distinct hydrophilic and hydrophobic regions, which during spray drying leads to its migration to the surface of droplets. ${ }^{7,8}$ This may result in formation of L-leucine rich shell around the drying droplet, interfering with the evaporation of water molecules, thus resulting in formation of corrugated particles. These particles have shown to display significantly reduced contact area and thereby, exhibit reduced cohesion and better dispersion. ${ }^{9}$ 
Recent studies have also indicated the importance of the role played by solid-state properties of L-leucine in reducing the cohesiveness of the spray-dried formulations. ${ }^{10}$ During drying of the droplet, L-leucine was found to crystallize early due to its limited water solubility and thereby gets accumulated at the surface of the droplet. Effectiveness of L-leucine in reducing the cohesivity of the formulations was thus related to the extent of crystallization of L-leucine in the evaporating droplet. ${ }^{11}$ Despite the above observation, the exact mechanism by which L-leucine reduces the surface energy of the spray-dried particles and influences particle morphology is still not clear. Additionally, the effect of co-spray drying with L-leucine on the physicochemical and material properties of spray-dried particles is not fully explored.

Another area which has received relatively little attention is the study of nature and extent of interaction between the inhaled drug particles and the pulmonary surfactant that represents the primary site of contact. ${ }^{12}$ Pulmonary surfactant plays a vital role in facilitating the effective pulmonary function and provides the medium for dissolution and consequentially uptake and transport of inhaled particles following deposition on the respiratory epithelia. Hence the study of interactions with the inhaled particles could provide further insights in developing better formulations.

The present study was undertaken to investigate the effect of L-leucine surface concentration on the physico-chemical properties, surface energy and Young's modulus of the composite particles containing voriconazole (VRZ). VRZ was chosen in a previous study published by our group where inhalable formulation of VRZ with L-leucine was developed for the treatment of invasive pulmonary aspergillosis. ${ }^{13}$ In addition, the effect of L-leucine on the interaction of composite particles with the pulmonary surfactant is also investigated. These insights could be helpful in developing inhalable formulations based on sound scientific principles, thus supporting "Quality by Design" approach. ${ }^{\mathbf{1 4}}$

\section{Materials and methods}

\subsection{Materials}

VRZ was obtained as the kind gift from Ranbaxy Laboratories (Gurgaon, India) and L-leucine was purchased from SigmaAldrich (Sydney, Australia). Dipalmitoylphosphatidylcholine (DDPC) was obtained from the Avanti Polar Lipids (Alabama, USA). All solvents and chemicals were of analytical grade and were supplied by Biolab (Victoria, Australia).

\subsection{Preparation of L-leucine modified VRZ microparticles}

VRZ and L-leucine in varying concentrations (Table 1) were dissolved in ethanol-water $(70: 30 \mathrm{v} / \mathrm{v})$ mixture in varying concentration (Sigma-Aldrich, Sydney, Australia) and spraydried using a Buchi Mini Spray Dryer B-290 coupled in series with a Buchi-296 dehumidifier and Buchi B-295 inert-loop (Buchi Laboratories, Flawil, Switzerland), using nitrogen as the drying gas to obtain L-leucine modified VRZ microparticles. The following spray dryer settings were used: feed concentration of $10 \mathrm{mg} \mathrm{ml}^{-1}$, inlet temperature $125{ }^{\circ} \mathrm{C}$, atomizer $700 \mathrm{~L}$ $\mathrm{h}^{-1}$, aspirator $40 \mathrm{~m}^{3} \mathrm{~h}^{-1}$ and feed rate $5 \%$. The corresponding outlet temperature obtained was $78{ }^{\circ} \mathrm{C}$.

\subsection{Morphology}

2.3.1 Scanning electron microscopy. The morphology of the microparticles was visualised using scanning electron microscopy (JEOL-6000 Benchtop, SEM, Tokyo, Japan) at $10 \mathrm{keV}$. Samples were mounted onto carbon sticky tapes, which were attached to aluminum stubs and coated (Smart coater, JEOL, Tokyo, Japan) with gold to $40 \mathrm{~nm}$ (coating thickness) prior to imaging.

2.3.2 Atomic force microscopy. PeakForce Tapping ${ }^{\mathrm{TM}}$ (PFT) AFM (Bruker, California, USA) was used to image the morphology of the spray-dried VRZ particles. Samples were mounted on glass slide with the help of two component epoxy resin (UHU, GmbH, D-Buhl) and imaged in air in tapping mode using AFM cantilever of spring constant $200 \mathrm{~N} \mathrm{~m}^{-1}$ (TAP525A, Bruker, USA). The images ( 512 by 512 pixels) were captured with a scan size of $2.0 \times 2.0 \mu \mathrm{m}$ at the scan rate of 0.5 lines per s. Images were analyzed with the help of NanoScope Analysis 1.5 version software (Bruker, California, USA).

\subsection{Particle size distribution}

Particle size distribution of spray-dried powders was measured by laser diffraction (Mastersizer 3000, Malvern Instruments, Worcs, UK) with dry powder feeder (Aeros S, Malvern Instruments, Worcs, UK). Approximately $5 \mathrm{mg}$ of sample was dispersed in air using 4 bar pressure and measured with a refractive index of 1.62 for VRZ. ${ }^{15}$ Samples were measured in triplicate.

\subsection{X-ray photoelectron spectroscopy (XPS)}

An XPS analysis method was applied to characterize quantitatively the extent of coverage of L-leucine on the VRZ particle surfaces. An AXIS Ultra Spectrometer with a monochromatic Al

Table 1 Composition and characteristics of L-leucine modified spray-dried VRZ inhalable dry powder. VRZ = voriconazole; LEU = leucine; data is represented as mean $\pm \mathrm{SD}(n=3)$

\begin{tabular}{|c|c|c|c|c|c|c|c|}
\hline Sample & Sample code & $\operatorname{VRZ}\left(\mathrm{mg} \mathrm{ml}^{-1}\right)$ & Leucine $\left(\mathrm{mg} \mathrm{ml}^{-1}\right)$ & Dv10 $(\mu \mathrm{m})$ & Dv50 $(\mu \mathrm{m})$ & Dv90 $(\mu \mathrm{m})$ & Span \\
\hline 1 & VRZ & 10.0 & 0.0 & $1.07 \pm 0.06$ & $4.52 \pm 0.07$ & $10.73 \pm 0.92$ & $2.13 \pm 0.15$ \\
\hline 2 & VRZ_LEU_10 & 9.0 & 1.0 & $0.51 \pm 0.02$ & $2.63 \pm 0.06$ & $7.07 \pm 0.33$ & $2.49 \pm 0.09$ \\
\hline 4 & VRZ_LEU_30 & 7.0 & 3.0 & $0.70 \pm 0.01$ & $2.31 \pm 0.09$ & $6.89 \pm 0.34$ & $2.69 \pm 0.04$ \\
\hline
\end{tabular}


K $\alpha$ source (Kratos Analytical Ltd., Manchester, UK) was used to determine the spectrum. A reference binding energy of $285.0 \mathrm{eV}$ was used for the aliphatic hydrocarbon C 1s component. Survey spectra (320 eV pass energy) using sensitivity factors provided by the manufacturer were used for the calculation of elemental composition. High-resolution spectra of each peak were recorded at $40 \mathrm{eV}$ pass energy with a peak width of typically $1.0-1.1 \mathrm{eV}$.

\subsection{Analysis of surface forces of L-leucine modified VRZ powders using colloid probes}

2.6.1 Preparation of drug probes. Particles of spray dried formulations were mounted onto the apex of tipless AFM cantilevers (N12 noAl, MikroScience, Brno, Czech) with the nominal spring constant of $0.65 \mathrm{~N} \mathrm{~m}^{-1}$ using a micromanipulation process as previously reported by Preuss and Butt. ${ }^{16}$ The probes were prepared by sticking a particle of the drug substance to the cantilever using a micromanipulator (MMO-203, Narishige, Japan). The cantilever was first dipped into twocomponent epoxy adhesive (UHU GmbH, D-Buhl) and afterwards moved to collect a particle from a glass plate. Evaluation of tip quality, quantity of glue and tip particle integrity prior to and post curing was verified using a light microscope (Axiotech Vario, Carl-Zeiss AG, D-Oberkochen). Prepared drug probe tips were stored in tightly sealed containers for $24 \mathrm{~h}$ prior to use.

2.6.2 Preparation of drug compact. Model drug compacts are widely used for the determination of interparticulate forces since they evade numerous issues that arise when measuring the adhesion between individual micronized particles. ${ }^{17-19}$ Model surfaces of spray-dried VRZ powders were prepared by direct compression. Approximately $30 \mathrm{mg}$ of spray-dried powder was weighed into a $6 \mathrm{~mm}$ die and compacted at a compression rate of $0.5 \mathrm{~mm} \mathrm{~s}^{-1}$ (700 kg; dwell time, $240 \mathrm{~s}$ ). Prepared model surfaces were stored in tightly sealed containers for $24 \mathrm{~h}$ prior to use. Extreme care was taken when handling the model surfaces to avoid contamination.

2.6.3 Measurement of surface force of spray-dried VRZ powders. Multiple force $v s$. distance curves were conducted between each drug probe and the corresponding compact disc of spray dried particle substrates using the AFM in the ForceVolume (FV) mode JPK NanoWizard I (JPK Instruments AG, Berlin, Germany). Individual force curves $(n=1024)$ were conducted over two distinct $100 \times 100 \mu \mathrm{m}$ areas using a $32 \times 32$ pixel raster. Force vs. distance curves were recorded at a frequency of $5 \mathrm{~Hz}$ maintaining a constant load during the measurements. The adhesion force was calculated using an inhouse software to evaluate cantilever deflection, which can be converted into force by using Hooke's law if the spring constant of the cantilever is known. ${ }^{20}$ The spring constant of the prepared drug probes was determined using the thermal noise method. ${ }^{21}$

\subsection{Effect of spray drying L-leucine on Young's modulus of the L-leucine modified VRZ microparticles}

Powders were compacted as previously discussed to obtain a relatively flat surface. Nanomechanical mapping was performed using the Dimension Icon ${ }^{\circledR}$ AFM (Bruker, MA, USA) in PeakForce Tapping mode with quantitative nanomechanical analysis
(QNM). Measurements were carried out with a silicon probe (spring constant approximately $200 \mathrm{~N} \mathrm{~m}^{-1}$, and of the radius of the tip rounding about $12 \mathrm{~nm}$; TAP525A, Bruker, USA). Cantilever deflection sensitivity were determined by ramping against the hard substrate (silicon wafer in our case). To determine spring constant and tip radius, an iterative process was adopted for estimating the force of indentation of cantilever $\left(F_{\text {tip }}\right)$ and cantilever tip radius $\left(R_{\text {tip }}\right)$ where the $F_{\text {tip }}$ was adjusted until the modulus matched the values for the standard polystyrene sample (PS) (Young's modulus 3-3.5 GPa). The adjusted $F_{\text {tip }}$ was then applied to the PS sample and the radius was again modified until the modulus matched the standard value. The iterative process was continued until the values of $F_{\text {tip }}$ and $R_{\text {tip }}$, which produced an exact match for PS with respect to the standard value, gave a mean modulus of PS that was within \pm 1 standard deviation of the standard value. Following this calibration process, the same applied force and same tip radius as well as the Poisson's ratio of 0.3 were used for all the AFM measurements of the remaining compact spray-dried powder surface. The line scan rate was equal to $0.5 \mathrm{~Hz}$ for the entire $256 \times 256$ pixels $(1 \times 1 \mu \mathrm{m})$ scan of the sample. Force-distance curves were extracted from the images of the compact surfaces of different spray-dried powders. These curves were then fitted with DMT model of particle contact and the Young's modulus values were determined for the different spray-dried formulations using the NanoScope Analysis 1.5 version software (Bruker, California, USA).

\subsection{Study of interaction between simulated pulmonary surfactant and L-leucine modified VRZ microparticles}

2.8.1 Preparation and visualization of dipalmitoylphosphatidylcholine (DPPC) monolayer. DPPC monolayers (simulated pulmonary surfactant) were produced using the Langmuir Blodgett (LB) approach (Model 2011, Nima Technology, Coventry, UK) according to previously published studies. ${ }^{\mathbf{1 2 , 2 2}}$ Briefly, a volume of $100 \mu \mathrm{l}$ DPPC solution in chloroform was applied to the air-water interface. A period of $10 \mathrm{~min}$ was allowed for the amphiphilic material to spread across the surface. LB pressure vs. area $(\pi-\mathrm{A})$ isotherms, (i.e. surface pressure as a function of molecular area at a constant temperature) were obtained by programming the trough barriers to move at $80 \mathrm{~cm}^{2} \mathrm{~min}^{-1}$ towards the Wilhelmy plate at the centre of the compartment. DPPC LB films were then produced by passing freshly cleaved muscovite mica at a $90^{\circ}$ angle through the DPPC LB monolayer at a constant surface pressure of $2 \mathrm{mN}$ $\mathrm{m}^{-1}$. Due to the hydrophilic nature of muscovite mica, DPPC monolayer deposition occurred when the solid support experienced an upstroke through the air-water interface; this process transformed the muscovite mica surface characteristic from hydrophilic to hydrophobic. A substrate transfer speed of 15 mm $\min ^{-1}$ was used. PeakForce Tapping ${ }^{\mathrm{TM}}$ (PFT) AFM (Bruker, California, USA) was utilized to visualize and confirm the formation of DPPC monolayer under ambient conditions with cantilevers of nominal spring constant $5 \mathrm{~N} \mathrm{~m}^{-1}$ (TAP150A, Bruker, USA). The scan size was $10 \mu \mathrm{m} \times 10 \mu \mathrm{m}$ whilst parameters such as the set point, gains and scan rate were varied to optimize image quality. 
2.8.2 Analysis of interaction between DPPC monolayer and drug probes. Five colloids probes were prepared for each of the VRZ and L-leucine modified formulations as mentioned in Section 2.6.1 and quantitative measurement of interaction between simulated pulmonary surfactant and the different formulations was carried out by force-volume measurements using JPK NanoWizard I (JPK Instruments AG, Berlin, Germany) over two separate areas of $100 \times 100 \mu \mathrm{m}$.

\subsection{Statistical analysis}

All results are expressed as mean \pm standard deviation of at least three separate determinants. One-way ANOVA followed by post hoc Tukey's test were performed to determine significance $(p<0.05)$ between treatment groups and control using GraphPad Prism 5 (GraphPad Software Inc, California, USA).

\section{Results and discussion}

The present research evaluated the effect of spray drying Lleucine on the surface energy and local elastic modulus of composite VRZ and L-leucine microparticles. In addition, the effect of L-leucine on the interaction of composite particles with the simulated pulmonary surfactant is also presented. In order to study these aspects, the spray-dried powders were first characterized for particle size, morphology and surface composition. Fig. 1 shows the chemical structure of voriconazole (VRZ) and L-leucine.

\subsection{Physicochemical characterization of inhalable powders}

The particle size distribution of the spray-dried VRZ powders is shown in Fig. 2 and the key size descriptors are listed in Table 1. Median volume diameter of the spray-dried VRZ formulations ranged from $2.31 \mu \mathrm{m}$ to $4.52 \mu \mathrm{m}$, suggesting that all formulations were of suitable size for inhalation delivery. Fig. 3 shows the representative scanning electron micrographs of the spray-dried formulations. When spray dried of its own (Fig. 3A) VRZ was found to present a plate like crystalline morphology. However, as the concentration of L-leucine was increased in the spray drying feedstock from $10 \% \mathrm{w} / \mathrm{w}$ to $30 \% \mathrm{w} / \mathrm{w}$, the particles were found to become more spherical and smaller in size (Fig. 3B-D). L-Leucine interfered with the crystallization of VRZ during the spray drying process (also seen by differential scanning calorimetry thermograms of inhalable powders, data not shown). The reduced particle sizes with increase L-leucine concentration was primarily due to reduced droplet size during the spray drying caused by the

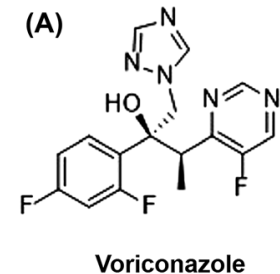

(B)<smiles>CC(C)C[C@H](N)C(=O)O</smiles>

L-Leucine

Fig. 1 Chemical structures of voriconazole (A) and L-leucine (B).

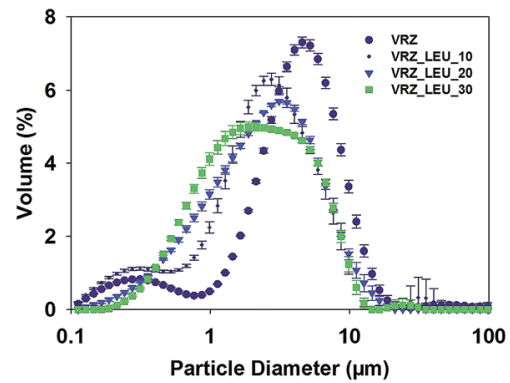

Fig. 2 Particle size distribution of the spray-dried VRZ and leucine modified VRZ formulations using laser diffraction $(n=3$; mean \pm SD).
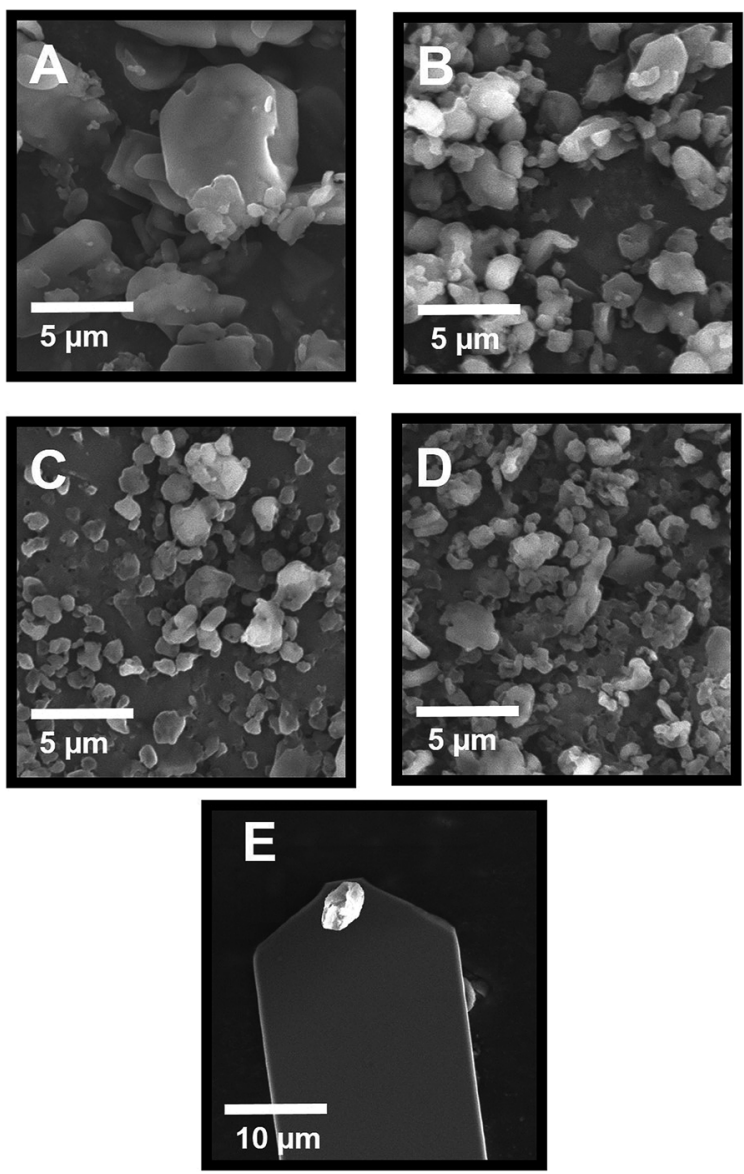

Fig. 3 Scanning electron micrographs of spray-dried VRZ (A), VRZ_LEU_10 (B), VRZ_LEU_20 (C) and VRZ_LEU_30 (D) and representative drug colloid probe (E).

lowering of the interfacial tension of the droplets due to the accumulation of L-leucine at the air-liquid interface caused by its relative hydrophobicity., ${ }^{\mathbf{9} 23}$ The sizes obtained from the morphological analysis were found to be in agreement with the size data obtained from laser diffraction.

Fig. 3E shows a representative SEM image of the colloid drug probe imaged after the AFM study. All the mounted drug particulates used in the AFM study exhibited an approximate size from 2-4 $\mu \mathrm{m}$ and clearly stood proud of the epoxy resin residue on the cantilever. Fig. 4A-D shows AFM topographical 

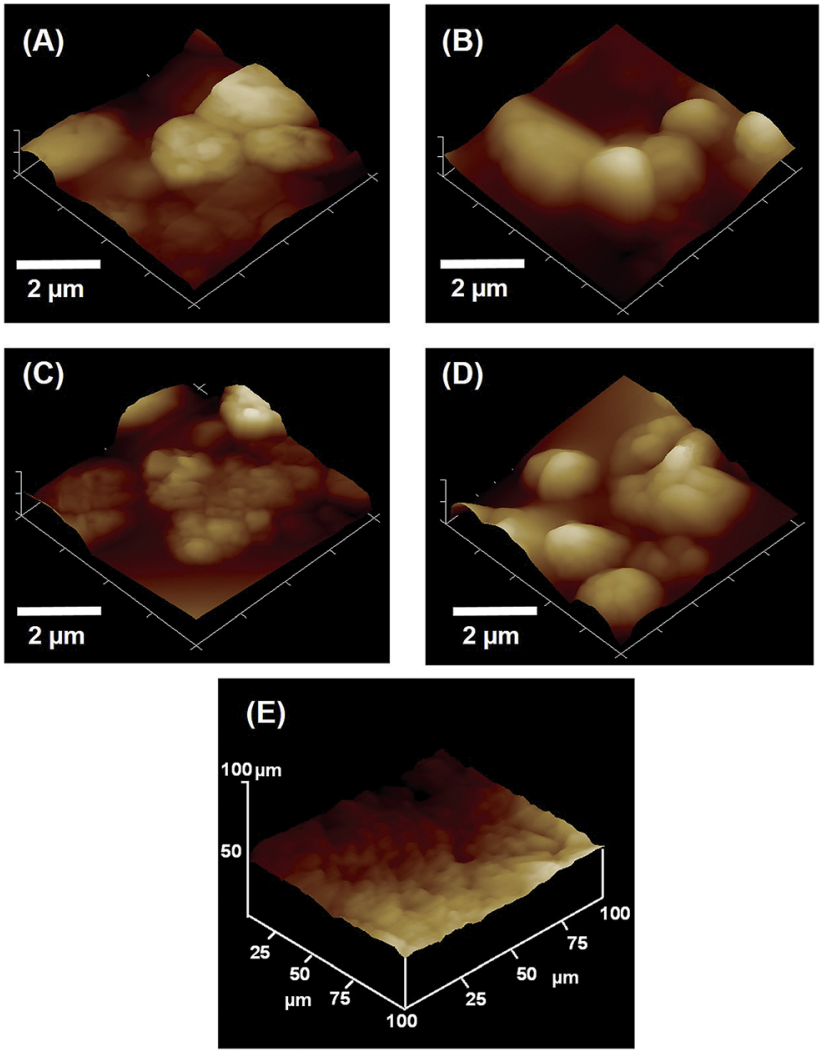

Fig. 4 Atomic force microscopy images of spray-dried VRZ (A), VRZ_LEU_10 (B), VRZ_LEU_20 (C) and VRZ_LEU_30 (D) and representative topographical image of drug compact surface $(X, Y$ and $Z=$ $100 \times 100 \times 100 \mu \mathrm{m})(\mathrm{E})$.

images of spray dried composite VRZ and L-leucine formulations. Particles exhibited rough, irregular morphology with size well in accordance with that observed with SEM and laser diffraction. Fig. 4E shows the representative AFM topographical image of the VRZ model compact employed to assess the cohesion force within the spray-dried VRZ powder. Similar compacts were also made for the other spray dried VRZ Lleucine powders to investigate the influence of L-leucine in modifying the cohesive balance within the developed formulations. Fig. 4E suggested an irregular surface topography of the model compact with the root mean square roughness $(100 \times$ $100 \mu \mathrm{m})$ of $89.2 \pm 13.2 \mathrm{~nm}(n=6)$. These values were quite similar in the order of magnitude as reported for the compacts from the previous AFM studies. ${ }^{19}$ However, it is worth mentioning that these values are dependent on the technique and methodology chosen.

\subsection{XPS analysis of the spray-dried powders}

Fig. 5A-D shows the XPS spectra of the spray dried surface modified VRZ powders, with Fig. 5E showing the surface chemical information of VRZ samples. XPS is highly surface sensitive technique due to the act that the photoelectrons are excited from the top 5-10 $\mathrm{nm}$ of the sample surface, hence the chemical properties measured here represent the outer most surface of the prepared particles. The signal from the element fluorine (F) can exclusively be attributed to the drug VRZ as there is no fluorine present in L-leucine, as depicted in the chemical structures of VRZ and L-leucine (Fig. 1). Concentration of element ' $F$ ' on the surface of spray dried surface modified VRZ particles decreased as the L-leucine concentration was increased from 0 to $30 \% \mathrm{w} / \mathrm{w}$ (Fig. $5 \mathrm{E}$ ). Spray drying of VRZ with L-leucine $(80: 20 \mathrm{w} / \mathrm{w})$ led to $95 \%$ surface coverage of the prepared particles with L-leucine as indicated by the decrease in concentration of ' $\mathrm{F}$ ' from $10.4 \%$ to $0.5 \%$ when $\mathrm{L}$-leucine was increased from 0 to $20 \% \mathrm{w} / \mathrm{w}$ (Fig. $5 \mathrm{E}$ ). Subsequent increase in the L-leucine concentration did not lead to any further increase in the surface coverage of L-leucine. These results clearly
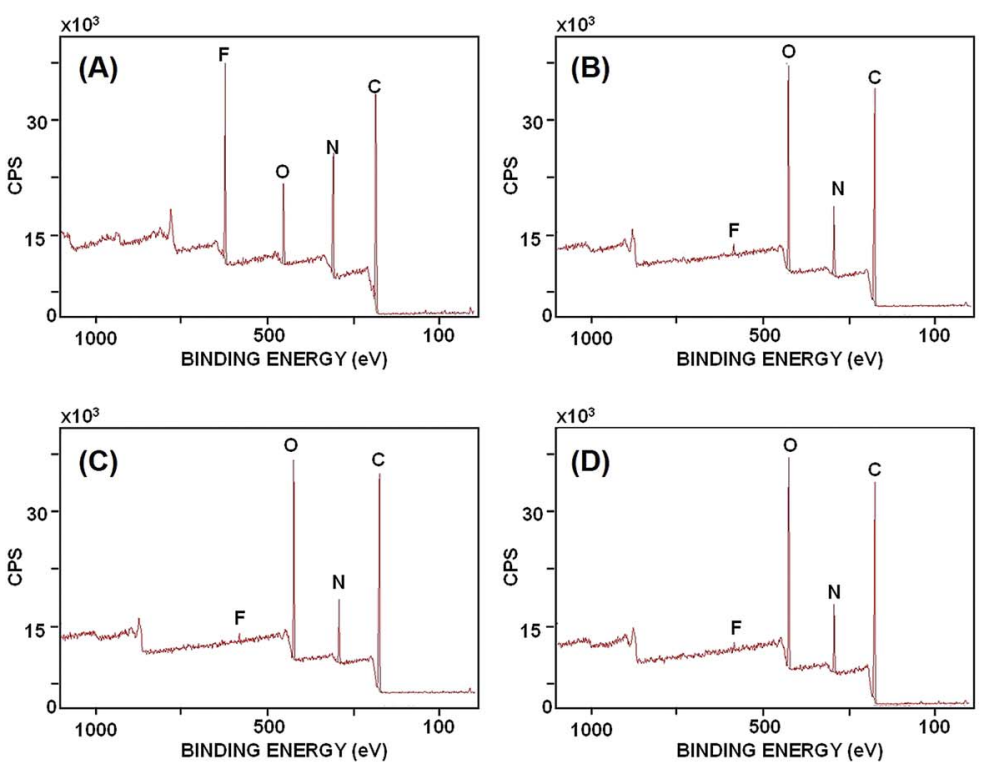

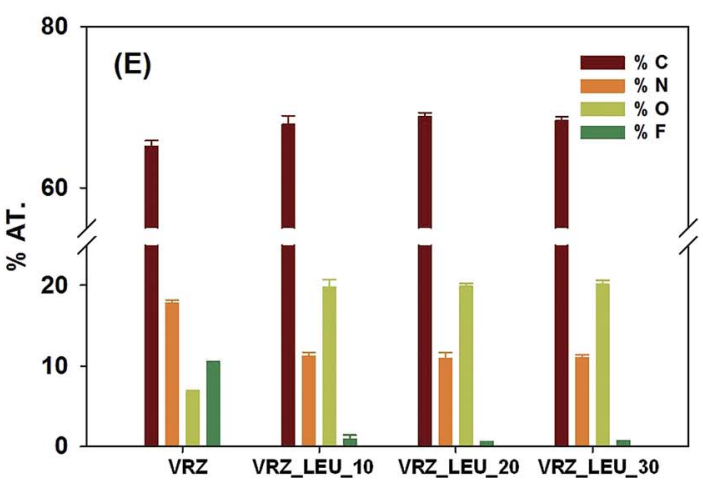

Fig. 5 Representative XPS spectra of spray-dried VRZ (A), VRZ_LEU_10 (B), VRZ_LEU_20 (C) and VRZ_LEU_30 (D) and relative percentage of carbon, nitrogen, oxygen and fluorine in the spray dried samples (E). 
indicate that the spray-dried VRZ powders are extensively covered by L-leucine when spray dried at $20 \% \mathrm{w} / \mathrm{W}$ or more $\mathrm{L}^{-}$ leucine concentration. The surface accumulation of $\mathrm{L}_{\text {-leucine }}$ can be explained by the existence of hydrophilic and hydrophobic domains within the L-leucine molecule which impart surfactant like properties to L-leucine. As a result, during the evaporation of droplet within the spray dryer, L-leucine tends to accumulate at the air/liquid interface, thus leading to its surface accumulation. ${ }^{9,24}$

\subsection{Effect of L-leucine in modifying the surface forces of spray-dried VRZ powders}

Multiple force-distance curves were conducted between each formulation probe and the corresponding compact disc of spray dried surface-modified VRZ powders substrates using AFM in the Force-Volume (FV) mode. Evaluation of all force distance curves measured over an $100 \times 100 \mu \mathrm{m}$ area $(n=1024)$ yielded a relatively wide distribution of cohesion force values with calculated standard deviations being of similar order as the mean. In addition, cohesion force histogram analysis showed that the data spread across more than one order of magnitude in an asymmetrical, positively skewed distribution. This is in accordance with previous studies on model compacts which have suggested lognormal cohesion force distributions for such positively skewed data. ${ }^{19,25}$

Therefore, the data from each drug probe was processed to generate cumulative-frequency log distribution. This distribution is demonstrated in Fig. 6 for different spray-dried VRZ powders for drug colloid probe 1 . The assumption of log normal distribution was further justified using full 4-parameter sigmoidal regression analysis, where $R^{2}$ values of $\geq 0.90$ was observed in all the cases (Table 2). Thus, a median value, or $50 \%$ cumulative undersize $\left(e_{0.5}\right)$, was chosen as the most appropriate descriptor for cohesion force measurements since the large positive skew in the data may adversely affect the mean values.

The effect of increasing concentrations of L-leucine in the spray drying feedstock on the $e_{0.5}$ is shown in Fig. 7 and Table 2 . In all formulations, a statistically significant $(p<0.05)$ decrease in the $e_{0.5}$ was observed with the increase in L-leucine concentration. This is also very well correlated $\left(R^{2}=0.996\right)$ with the increase in the fine particle fraction (\% aerosol $<5 \mu \mathrm{m})$ of the spray dried formulations (data not shown), as the concentration

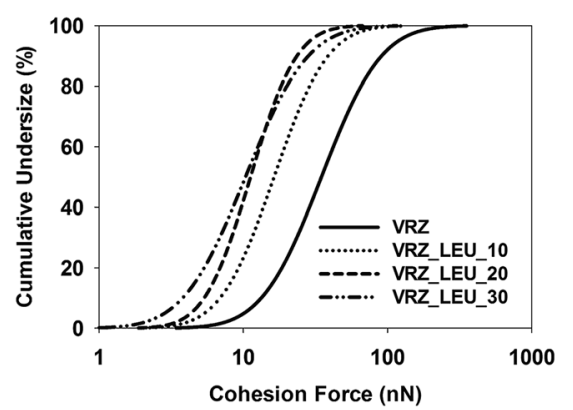

Fig. 6 Representative cohesion force distribution of different spray dried VRZ inhalable powders for drug colloid probe 1.
Table 2 Spray dried VRZ formulations median cohesion force values for five different colloid probes

\begin{tabular}{|c|c|c|c|c|c|}
\hline \multirow[b]{2}{*}{ Tip } & \multirow[b]{2}{*}{ Parameter } & \multicolumn{4}{|c|}{ Formulation } \\
\hline & & VRZ & VRZ_LEU_10 & VRZ_LEU_20 & VRZ_LEU_30 \\
\hline 1 & $e_{0.5}(\mathrm{nN})^{a}$ & 34.81 & 16.35 & 11.23 & 10.36 \\
\hline 1 & $\mathrm{GSD}^{b}$ & 2.12 & 1.96 & 1.98 & 2.18 \\
\hline 1 & $R^{2 c}$ & 0.906 & 0.916 & 0.923 & 0.908 \\
\hline 2 & $e_{0.5}(\mathrm{nN})^{a}$ & 49.14 & 17.59 & 10.89 & 10.24 \\
\hline 2 & $\mathrm{GSD}^{b}$ & 2.17 & 1.94 & 1.71 & 1.90 \\
\hline 2 & $R^{2 c}$ & 0.904 & 0.917 & 0.930 & 0.92 \\
\hline 3 & $e_{0.5}(\mathrm{nN})^{a}$ & 42.96 & 13.94 & 10.88 & 16.92 \\
\hline 3 & $\mathrm{GSD}^{b}$ & 2.08 & 1.78 & 1.56 & 1.49 \\
\hline 3 & $R^{2 c}$ & 0.908 & 0.926 & 0.938 & 0.942 \\
\hline 4 & $e_{0.5}(\mathrm{nN})^{a}$ & 30.20 & 12.88 & 11.72 & 14.64 \\
\hline 4 & $\mathrm{GSD}^{b}$ & 2.22 & 2.17 & 1.71 & 1.98 \\
\hline 4 & $R^{2 c}$ & 0.901 & 0.904 & 0.930 & 0.915 \\
\hline 5 & $e_{0.5}(\mathrm{nN})^{a}$ & 29.90 & 17.23 & 13.59 & 10.08 \\
\hline 5 & $\mathrm{GSD}^{b}$ & 2.05 & 1.75 & 1.64 & 2.02 \\
\hline 5 & $R^{2 c}$ & 0.91 & 0.928 & 0.934 & 0.912 \\
\hline
\end{tabular}

${ }^{a} e_{0.5}$, median cohesion force ( $n=1024$ for each of 5 colloid probes). ${ }^{b}$ Geometric standard deviation $\left(e_{0.841} / e_{0.159}\right)^{0.5} \cdot{ }^{c} R^{2}$, full 4-parameter sigmoidal regression analysis of cumulative undersize (\%) vs. cohesion force value plot.

of L-leucine was increased in these formulations. Thus, the ability of L-leucine to modify the cohesive forces within the spray-dried formulations could be attributed as the prime reason for the improvement in the aerosolization properties of these formulations. This observation was in agreement with the previously reported studies. ${ }^{2,24,26}$

\subsection{Effect of co-spray drying of L-leucine on the Young's modulus of the inhalable powders}

Local mechanical properties of the drug products play a very important role in regulating the material interactions as well as the ability to withstand stresses during the transportation and powder filling. ${ }^{27,28}$ AFM-based nanomechanical characterization using Bruker's Peak Force ${ }^{\circledR}$ Tapping mode helps to identify

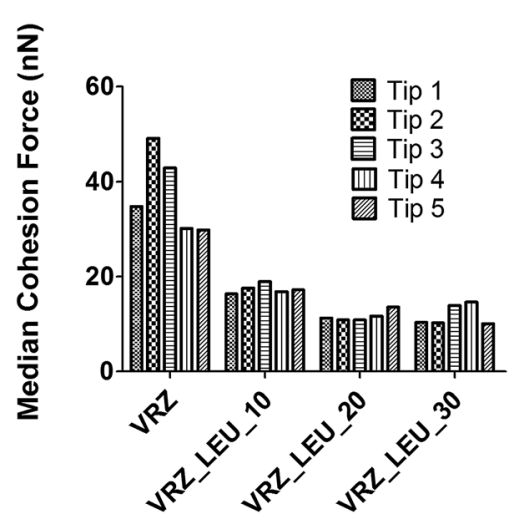

Fig. $7\left(e_{0.5}\right)$ median cohesion force values for spray dried surface modified VRZ drug probes on respective model drug compacts ( $n=$ 1024). 
material variations unambiguously and at high resolution across a topographic image. Determination of local elastic properties of inhalable dry powders would help to determine how particulates may interact and respond to processing that cause deformation during manufacturing or delivery. In this study an investigation was undertaken to assess whether cospray drying of L-leucine along with VRZ had an effect in altering the young's modulus of composite particles.

Calibration of AFM tip radius $(R)$ and applied tip-sample force $\left(F_{\text {tip }}\right)$ was were performed on standard polystyrene sample as described in the Section 2.6 yielding Young's moduli of the standard polystyrene sample of $3.12 \pm 0.45 \mathrm{GPa}$ which is in close agreement with the standard value of $3 \mathrm{GPa}$ as supplied by manufacturer. This resulted in the experimental settings of $12 \mathrm{~nm}$ of tip radius and $40 \mathrm{nN}$ of applied force.

Fig. 8 shows the high-resolution quantitative nanomechanical maps of different compacts of formulations obtained by the Peak Force Tapping mode. A statistical quantitative comparison of average Young's modulus of different samples is shown in Fig. 9. Calculation of average values was performed by extracting the force curves over three

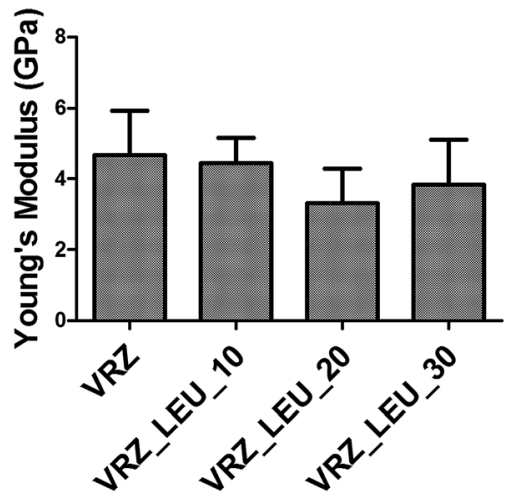

Fig. 9 Young's modulus values of spray dried VRZ inhalable powders obtained by extracting force distance curves from the elasticity images obtained by Peak Force Tapping.

different positions on Peak Force Tapping high-resolution nanomechanical maps. The retract curve was fitted using the Derjaguin-Muller-Toporov (DMT) model. ${ }^{29}$ Young's modulus values did not differ significantly $(p>0.05)$ between the

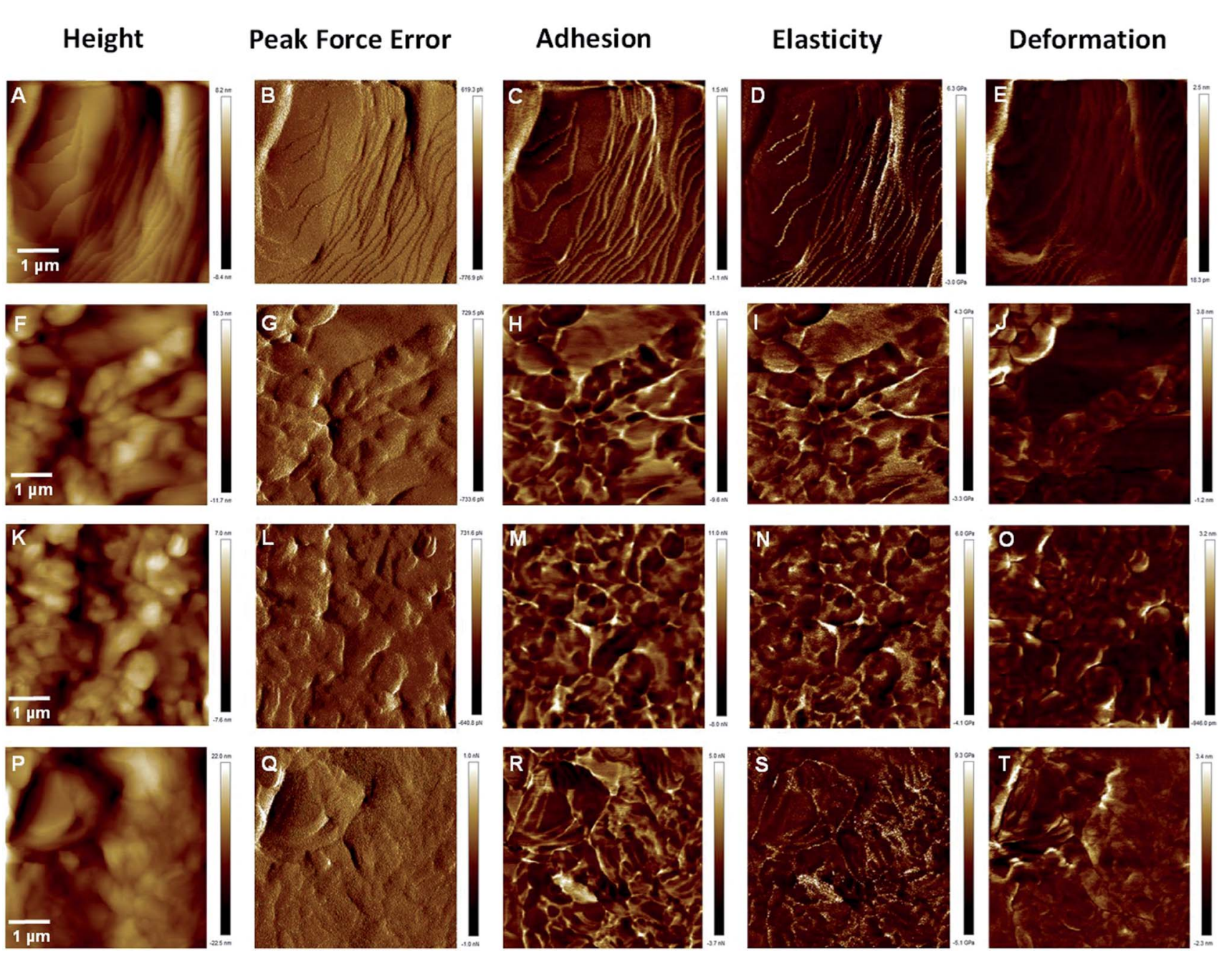

Fig. 8 Height, peak force error, adhesion, elasticity and deformation mapping of compacts of spray dried VRZ alone (A-E), VRZ_LEU_10 (FJ),VRZ_LEU_20 (K-O) and VRZ_LEU_30 (P-T) using Peak Force Tapping. 
different formulations and ranged between 3.32 to $4.67 \mathrm{GPa}$ for all the formulations. This clearly demonstrated that co-spray drying of L-leucine (up to $30 \% \mathrm{w} / \mathrm{w}$ ) with VRZ did not lead to significant change in the Young's modulus value of the composite particles. This is considered to be desirable as composite particles retain sufficient strength to withstand the stress or deformation during the powder manufacturing, transport and handling.

\subsection{AFM drug particle-pulmonary surfactant interaction}

There are very few studies where the physical interactions of inhaled particles with pulmonary surfactant have been investigated. The aim of this study was to investigate whether the increase in L-leucine content in the composite particles changed the nature and extent of physical interaction with simulated pulmonary surfactant.

The Langmuir Blodgett (LB) approach can be utilised for the formation of phospholipid-based monomolecular films, representative of that found in the deep lungs. ${ }^{12,30-32}$ DPPC was used for the formation of lipid monolayer as it constitutes half of pulmonary surfactant phospholipids. ${ }^{33}$ Prior to the formation of DPPC monolayer, the LB trough was calibrated for surface pressure measurements using arachidic acid, which showed a characteristic kink at $25.6 \mathrm{mN} \mathrm{m}^{-1}$ (Fig. 10A).

A typical DPPC LB $\pi-A$ isotherm along with corresponding film morphology, at specific surface pressure, is presented in Fig. 10A-F. The DPPC LB $\pi-\mathrm{A}$ isotherm exhibited a plateau at approximately $2 \mathrm{mN} \mathrm{m}^{-1}$ and the collapse pressure point was in the region of $50 \mathrm{mN} \mathrm{m}^{-1}$, in agreement with previously reported data. ${ }^{1,34}$ As the area decreases and surface pressure increases, DPPC monolayer undergoes phase transition that followed: liquid expansion (initial slope region); coexistence of the liquidcondensed and liquid-expanded phases (plateau region) and liquid-condensed phase (LC) (steep slope region). Monolayers of DPPC were formed on the muscovite mica at constant surface pressure of $2 \mathrm{mN} \mathrm{m}^{-1}$. At this pressure, liquid-condensed and liquid-expanded phases of DPPC coexist. Pulmonary surfactant, at physiological temperature, is well known to exist in semicrystalline solid phase (liquid-condensed phase) and a disordered fluid phase (liquid-expanded phase) at the air-liquid interface. ${ }^{34-36}$ In order to confirm the formation of DPPC monolayers on the mica surface, AFM images of the coated mica surface was carried out. A smooth homogeneous surface was found. To proof that the mica was indeed covered by a DPPC monolayer, a wear test was performed by scanning an area of $500 \mathrm{~nm} \times 500 \mathrm{~nm}$ in contact mode at high load. The surface was then again imaged at low load and the accumulation of deposited lipids along the edges of the scratched area was observed, confirming the formation of DPPC monolayer on the muscovite mica (Fig. 10C-F).

AFM adhesion data relating to the interaction between the $\mathrm{L}^{-}$ leucine modified VRZ microparticles and simulated pulmonary surfactant DPPC LB films under ambient condition is presented in Fig. 10G. Results clearly suggested a decrease in interaction between the particles and DPPC monolayers with increase in concentration of $\mathrm{L}$-leucine $(\mathrm{w} / \mathrm{w})$ in the composite particles. The observed trend in the adhesion measurements may be ascribed to several factors such as particle surface chemistry, surface energetics of the composite particles and as well as the contact geometry of the interacting surfaces. Overall it was anticipated that the exposed hydrophilicity of the composite particles, due to the surface coverage by L-leucine as determined by XPS, would dominate the interaction. ${ }^{24,37}$

DPPC monolayer when transferred on muscovite mica using LB trough will contain phospholipid chains that are well ordered and oriented towards the external environment. This results in a hydrophobic cover comprising of terminal methyl groups linked with fatty acid acyl chains. ${ }^{\mathbf{1 , 1 2}}$ Repulsive interactions are generally known to operate between the hydrophobic and
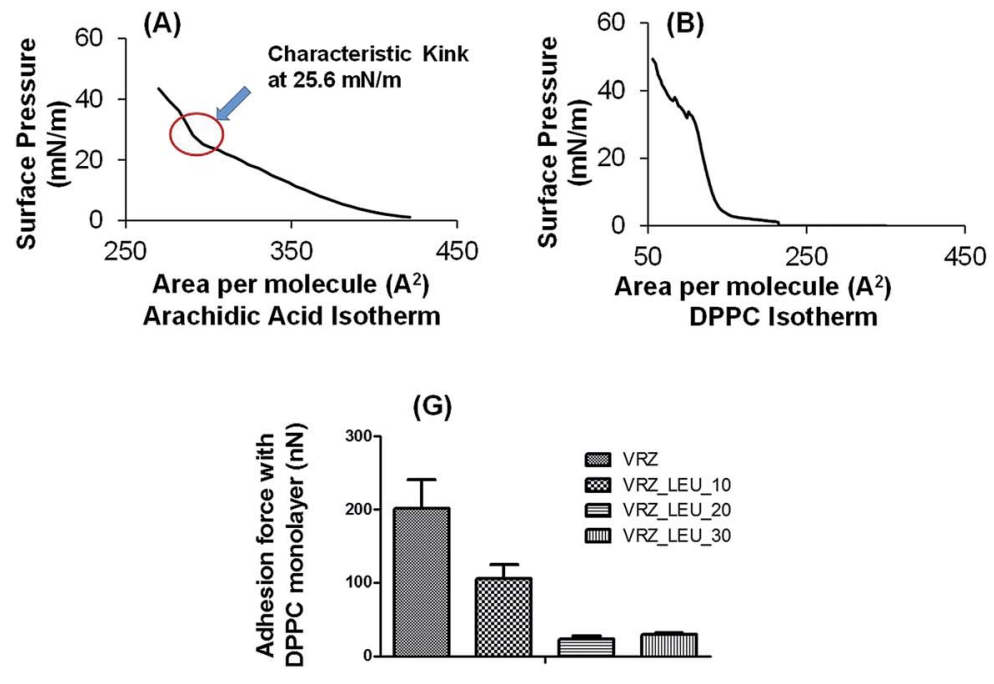

(G)

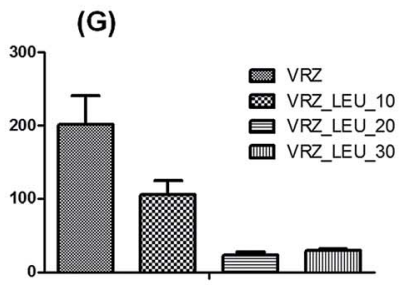

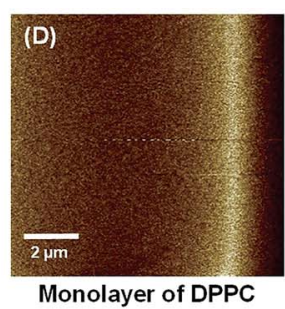
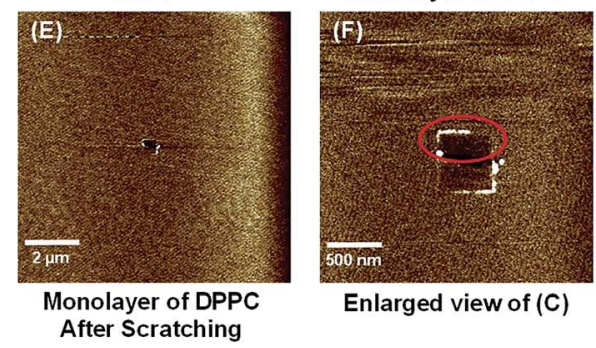

Enlarged view of (C)

Fig. $10 \mathrm{~A} \pi-\mathrm{A}$ isotherm at $25^{\circ} \mathrm{C}$ of arachidic acid (A) and DPPC (B); monolayers at an air-water interface; AFM topographical images of bare mica (C) and DPPC monolayers (D-F); AFM adhesion data relating to the interaction between the L-leucine modified VRZ powders and DPPC LB films under ambient conditions (G). 
hydrophilic surfaces, however, the exact nature of interactions depends upon type of interacting surfaces and the aqueous medium in-between. ${ }^{38,39}$ Increase in L-leucine concentration results in increase in surface hydrophilicity of the composite particles and hence increased repulsion between the simulated pulmonary surfactant and the composite particles was observed and as a consequence interaction was decreased. In addition, we also assume contact geometry between the composite particles and the pulmonary surfactant may also have influenced the extent of the physical interaction. ${ }^{40} \mathrm{VRZ}$, when spray dried alone, resulted in irregular shaped particles that may have resulted in multiple contacts with DPPC monolayers and thereby, exhibited greater interaction. On the contrary, when VRZ was spray dried with increasing concentrations of L-leucine, the particle morphology was found to become more spherical, thus resulting in lesser degree of contact with the DPPC monolayer, further adding to the decreased interaction between the composite particles and DPPC monolayer. This study indicates that the $\mathrm{L}^{-}$ leucine has potential in altering the interactions between VRZ and pulmonary surfactant.

\section{Conclusions}

AFM holds great potential in the quantification of cohesion/ adhesion forces between the two interacting surfaces. The present investigation highlights the effect of surface L-leucine concentration on particle morphology, surface energy and Young's modulus of composite particles, as well as interaction with the simulated pulmonary surfactant. This study has substantially clarified that increase in surface concentration of L-leucine effectively reduced the cohesive forces within formulation and increase in surface hydrophilicity of the composite particles led to reduced interaction with the simulated pulmonary surfactant. However, co-spraying of L-leucine with VRZ did not significantly influence the Young's modulus of the composite particles. This understanding can be employed in future studies to optimize the performance of inhalable spraydried formulations.

\section{Acknowledgements}

Authors are thankful to Director, NIPER, Woolcock Institute of Medical Research and Max Planck Institute for Polymer Research for providing necessary infrastructure facilities. SA is the recipient of an Endeavour Research Fellowship and German Academic Exchange Service (DAAD) Scholarship from the Australian and German government, respectively, in 2014 and the work was carried out as a part of these fellowships. The authors have no other relevant affiliations or financial involvement with any organisation or entity with a financial interest in or financial conflict with the subject matter or materials discussed in the manuscript apart from those disclosed.

\section{References}

1 G. P. Borissevitch, M. Tabak and O. N. Oliveira, Biochim. Biophys. Acta, 1996, 1278, 12-18.
2 Q. T. Zhou, S. S. Leung, P. Tang, T. Parumasivam, Z. H. Loh and H. K. Chan, Adv. Drug Delivery Rev., 2015, 85, 83-99.

3 S. W. Stein, P. Sheth, P. D. Hodson and P. B. Myrdal, AAPS PharmSciTech, 2014, 15, 326-338.

4 S. Claus, C. Weiler, J. Schiewe and W. Friess, Eur. J. Pharm. Biopharm., 2014, 86, 1-6.

5 Q. T. Zhou, L. Qu, I. Larson, P. J. Stewart and D. A. Morton, Int. J. Pharm., 2010, 394, 50-59.

6 K. Kendall and C. Stainton, Powder Technol., 2001, 121, 223229.

7 C. Gervelas, A. L. Serandour, S. Geiger, G. Grillon, P. Fritsch, C. Taulelle, B. Le Gall, H. Benech, J. R. Deverre, E. Fattal and N. Tsapis, J. Controlled Release, 2007, 118, 78-86.

8 J. G. Chan, A. S. Tyne, A. Pang, H. K. Chan, P. M. Young, W. J. Britton, C. C. Duke and D. Traini, Pharm. Res., 2014, 31, 1239-1253.

9 L. Cruz, E. Fattal, L. Tasso, G. C. Freitas, A. B. Carregaro, S. S. Guterres, A. R. Pohlmann and N. Tsapis, J. Controlled Release, 2011, 152, 370-375.

10 R. Vehring, Pharm. Res., 2008, 25, 999-1022.

11 A. L. Feng, M. A. Boraey, M. A. Gwin, P. R. Finlay, P. J. Kuehl and R. Vehring, Int. J. Pharm., 2011, 409, 156-163.

12 M. J. Davies, A. Brindley, X. Chen, S. W. Doughty, M. Marlow and C. J. Roberts, Colloids Surf., B, 2009, 73, 97-102.

13 S. Arora, M. Haghi, P. M. Young, M. Kappl, D. Traini and S. Jain, Expert Opin. Drug Delivery, 2015, 1-11.

14 S. Arora, R. R. Mahajan, V. Kushwah, D. Baradia, A. Misra and S. Jain, RSC Adv., 2015, 5, 38030-38043.

15 S. Arora, M. Haghi, C. Y. Loo, D. Traini, P. M. Young and S. Jain, Mol. Pharm., 2015, 12, 2001-2009.

16 M. Preuss and H.-J. Butt, Langmuir, 1998, 14, 3164-3174.

17 F. Podczeck, J. M. Newton and M. B. James, J. Colloid Interface Sci., 1997, 187, 484-491.

18 F. Podczeck, J. M. Newton and M. B. James, Int. J. Pharm., 1997, 149, 151-160.

19 P. M. Young, R. Price, M. J. Tobyn, M. Buttrum and F. Dey, J. Pharm. Sci., 2003, 92, 815-822.

20 M. Lohrmann, M. Kappl, H. J. Butt, N. A. Urbanetz and B. C. Lippold, Eur. J. Pharm. Biopharm., 2007, 67, 579-586.

21 J. L. Hutter and J. Bechhoefer, Rev. Sci. Instrum., 1993, 64, 1868-1873.

22 M. H. Kopf, H. Harder, J. Reiche and S. Santer, Langmuir, 2011, 27, 12354-12360.

23 N. R. Rabbani and P. C. Seville, J. Controlled Release, 2005, 110, 130-140.

24 N. Y. Chew, B. Y. Shekunov, H. H. Tong, A. H. Chow, C. Savage, J. Wu and H. K. Chan, J. Pharm. Sci., 2005, 94, 2289-2300.

25 P. M. Young, M. J. Tobyn, R. Price, M. Buttrum and F. Dey, J. Pharm. Sci., 2006, 95, 1800-1809.

26 J. Fiegel, L. Garcia-Contreras, M. Thomas, J. VerBerkmoes, K. Elbert, A. Hickey and D. Edwards, Pharm. Res., 2008, 25, 805-811.

27 M. Davies, A. Brindley, X. Chen, M. Marlow, S. W. Doughty, I. Shrubb and C. J. Roberts, Pharm. Res., 2005, 22, 1158-1166. 28 R. Roberts and R. Rowe, Int. J. Pharm., 1987, 37, 15-18. 
29 B. V. Derjaguin, V. M. Muller and Y. P. Toporov, J. Colloid Interface Sci., 1975, 53, 314-326.

30 J. A. Zasadzinski, R. Viswanathan, L. Madsen, J. Garnaes and D. K. Schwartz, Science, 1994, 263, 1726-1733.

31 G. Brezesinski and H. Mohwald, Adv. Colloid Interface Sci., 2003, 100-102, 563-584.

32 S. Garcia-Manyes, O. Domenech, F. Sanz, M. T. Montero and J. Hernandez-Borrell, Biochim. Biophys. Acta, 2007, 1768, 1190-1198.

33 J. Perez-Gil, Biochim. Biophys. Acta, 2008, 1778, 1676-1695.

34 S. L. Duncan and R. G. Larson, Biophys. J., 2008, 94, 29652986.

35 C. Casals and O. Canadas, Biochim. Biophys. Acta, 2012, 1818, 2550-2562.
36 B. Piknova, V. Schram and S. B. Hall, Curr. Opin. Struct. Biol., 2002, 12, 487-494.

37 S. Mangal, F. Meiser, G. Tan, T. Gengenbach, J. Denman, M. R. Rowles, I. Larson and D. A. Morton, Eur. J. Pharm. Biopharm., 2015, 94, 160-169.

38 A. Faghihnejad and H. Zeng, Langmuir, 2013, 29, 1244312451.

39 E. Kokkoli and C. F. Zukoski, J. Colloid Interface Sci., 2000, 230, 176-180.

40 J. C. Hooton, C. S. German, S. Allen, M. C. Davies, C. J. Roberts, S. J. Tendler and P. M. Williams, Pharm. Res., 2004, 21, 953-961. 